\title{
Wildauer Absolventinnen auf dem Arbeitsmarkt Ergebnisse einer Befragung
}

\author{
Michael Laschke, Brigitte Lindert
}

\begin{abstract}
Historie
Im Herbst 1997 fanden Absolventinnen der Ingenieurschule Wildau und der Technischen Fachhochschule Wildau einen Fragebogen ihrer Schule im Briefkasten. Das Anliegen dieser Befragung war die Sammlung von Daten zur Integration der Absolventinnen der Wildauer Schulen auf dem Arbeitsmarkt im Zusammenhang mit einem geplanten Foschungsprojekt zum Thema „Frauen und Technik“.

Das geplante Gesamtprojekt greift die in den Wirtschafts- und Sozialwissenschaften seit langem kontroverse Diskussion auf, ob der Trend der Integration von Frauen in die Erwerbstätigkeit fortgesetzt werden kann oder ob Frauen in noch stärkerem Maße als Männer zu den Verliererinnen betrieblicher Rationalisierung und gesellschaftlicher Umverteilung zwischen bezahlter und unbezahlter Arbeit werden.
\end{abstract}

Neue Aspekte in dieser Diskussion werden einerseits durch solche technischen Entwicklungsrichtungen gesehen, die die Automatisierung von Routinetätigkeiten und eine weitere Flexibilisierung in der Produktion und in den Dienstleistungen technisch möglich machen und gleichzeitig spezielle Anforderungen an die Mitarbeiter, wie soziale Kompetenz, Kommunikations- und Teamfähigkeit sowie vernetztes Denken hervorbringen. Arbeitsanforderungen und -bedingungen also, die für Frauen besonders geeignet erscheinen. ${ }^{*}$ Andererseits werden die mit den Globalisierungsprozessen verbundenen Verlagerungen von Arbeitsplätzen und die Veränderungen in den Arbeitsbedingungen als Faktoren betrachtet, die die Einbindung von Frauen in den Arbeitsmarkt erschweren.

Gleichzeitig rufen diese neuen technischen Möglichkeiten einen verstärkten Druck auf die Verschlankung der Produktion und des Managements hervor. Der Kostendruck, der damit verbunden ist, wird gleichermaßen auf den menschlichen Faktor übertragen. Investitionen in das Humankapital werden sehr häufig noch immer als Kosten und nicht als Chance verstanden.

Auch wenn allgemein anerkannt wird, daß die Potentiale neuer Techniken nur bei Vorhandensein von qualifizierten, motivierten und leistungsbereiten Mitarbeiter/-innen voll durchgesetzt werden können, wobei hierbei akademisch gebildete Frauen hinsichtlich Motivation und Leistungsbereitschaft gegenüber Männern oft günstiger beurteilt werden, ist nicht zu leugnen, daß die Chancen akademisch gebildeter Frauen auf dem Arbeitsmarkt nicht zum Besten bestellt sind. Mit der Vereinigung Deutschlands hat sich diese Lage keinesfalls gebessert. Im Gegenteil: Angesichts der gegenwärtigen Arbeitsmarktlage häufen sich die Aussagen, die die hohe „Erwerbsneigung“ von Frauen besonders in den „neuen“ Bundesländern ausgesprochen kritisch sehen und ihnen Tätigkeitsfelder außerhalb des Einkommenserwerbs empfehlen. Für das Beherrschen künftiger technischer Entwicklungen ist eine solche Auffassung ausgesprochen kontraproduktiv.

Es kommt vielmehr darauf an, die Anforderungen an die wissenschaftliche Qualifikation mit diesen langfristigen Tendenzen der technischen und wirtschaftlichen $\mathrm{Ar}$ beitsbedingungen, mit dem Herausbilden neuer $\mathrm{Ar}$ beitsmarktsegmente zu verknüpfen. Das Projekt soll dafür neue Möglichkeiten aufzeigen. Die begonnene Analyse der Beschäftigungsverhältnisse von Wildauer Absolventinnen ist hierin einzuordnen.

\section{Ausgangssituation}

Die Ausgangssituation ist durch folgende Zahlen aus den Unterlagen der Fachhochschule Wildau gekennzeichnet. Von 1984 bis 1997 meldeten insgesamt 2262 Personen ihr Studieninteresse an. Darunter befanden sich 737 Frauen. Von den Studieninteressenten und Zugelassenen traten 635, unter ihnen 178 Frauen, das Studium nicht an bzw. brachen es vorzeitig aus den verschiedensten Ursachen ab.

Studierende, die das Studium begannen und nicht abbrachen, wurden als „erfolgreich Studierende" betrachtet, ohne damit eine inhaltliche Aussage über das erreichte Ergebnis vornehmen zu wollen. „Erfolgreich Studierende“ waren damit 1627 Studenten, unter ihnen 559 Frauen. Ca. 245 von ihnen studieren noch, standen also für die Untersuchung nicht zur Verfügung. Wir gingen davon aus, daß dies alle Direktstudenten der Immatrikulationsjahrgänge ab 1994 betrifft, die nicht in Sonderstudienformen eingegliedert sind.

Über den Gesamtzeitraum betrachtet, betrug der Anteil der Frauen an den Bewerbungen zum Studium am Hochschulstandort Wildau etwas weniger als ein Drittel. Ihr Anteil am Nichtantritt bzw. an der vorzeitigen Beendigung des Studiums lag im Durchschnitt der Gesamtbewerbungen.

\section{Gründung der Technischen Fachhochschule Wildau}

Die Vereinigung der beiden deutschen Staaten brachte einen erheblichen Umbruch in den Lebenssituationen und in den Erwerbsbedingungen der ostdeutschen Bevölkerung. Das Bildungssystem wurde neu strukturiert. Studienanmeldungen und die erfolgreich Studierenden 
an der am 22.10.1991 neugegründeten Technischen Fachhochschule Wildau zeigt Tabelle 1.

TABELLE 1: Erfolgreich Studierende nach Zeitabschnitten

1984-1990 1991-1997

\begin{tabular}{|c|c|c|}
\hline \multicolumn{3}{|l|}{ Anmeldungen } \\
\hline gesamt & 814 & 1448 \\
\hline darunter Frauen & 272 & 465 \\
\hline Anteil Frauen Prozent & 33,4 & 32,1 \\
\hline \multicolumn{3}{|l|}{ Abbrecher } \\
\hline gesamt & 262 & 373 \\
\hline darunter Frauen & 90 & 88 \\
\hline $\begin{array}{l}\text { Anteil Abbrecher an } \\
\text { Anmeldungen gesamt }\end{array}$ & 32,1 & 25,7 \\
\hline $\begin{array}{l}\text { Anteil der Frauen an den Ab- } \\
\text { brechern gesamt Prozent }\end{array}$ & 34,3 & 23,5 \\
\hline $\begin{array}{l}\text { Anteil der weiblichen } \\
\text { Abbrecher an weiblichen }\end{array}$ & & \\
\hline Anmeldungen Prozent & 33,0 & 18,9 \\
\hline \multicolumn{3}{|l|}{ Erfolgreich Studierende } \\
\hline gesamt & 552 & 1075 \\
\hline darunter Frauen & 182 & 377 \\
\hline $\begin{array}{l}\text { Anteil der Frauen an den } \\
\text { erfolgreich Studierenden } \\
\text { Prozent }\end{array}$ & 32,9 & 35,0 \\
\hline
\end{tabular}

Quelle: Errechnet nach Unterlagen der TFHW

Aus der Tabelle 1 ergibt sich ein deutlicher Anstieg der Studienanmeldungen im Zeitraum nach 1991, wobei der Anteil der Frauen im Vergleich zum Zeitraum 1984 bis 1990 leicht zurückging.

Allerdings korrigierten die Frauen, die sich für ein Studium angemeldet hatten, diese Entscheidung in deutlich geringerem Ausmaß als vor 1990.

Der Anteil der weiblichen Abbrecher an den weiblichen Anmeldungen sank von 33 Prozent 1984 bis 1990 auf 18 Prozent von 1991 bis 1997.

Daraus ist zu schlußfolgern, daß die Entscheidung für ein Studium an der Technischen Fachhochschule Wildau von Frauen mit größerer Konsequenz „durchgezogen“ wird. Inwieweit sich dahinter ein deutlicher Motivationsschub bei den weiblichen Studierenden verbirgt, angesichts des erleichterten Zugangs zu den höheren Schulen die berufliche Qualifikation zu vervollkommnen, Chancengleichheit also bewußt zu nutzen oder (insbesondere bei den Teilnehmerinnen an den Brückenkursen) der Zwang, einen anerkannten Studienabschluß zu erwerben, bleibt zu hinterfragen.

\section{Fachrichłungsstruktur weiblicher Studierender}

Die fachrichtungsbezogenen Studieninteressen verschoben sich mit den gesellschaftlichen Umbrüchen 1989/ 1990 in den ostdeutschen Ländern. Allgemein wird ein
Rückgang weiblicher Studierender in den technischen Fachrichtungen beobachtet. Dieser Rückgang wurde auch an der Technischen Fachhochschule Wildau spürbar, wie in Graphik 2 dargestellt ist. Sie zeigt die Anzahl der Studierenden in den technischen Richtungen in den Zeitabschnitten vor 1990 und ab 1991. Wegen der geringen Zahlen weiblicher Studierender in einzelnen Studiengängen ab 1991 erfolgte eine Zusammenfassung der Verfahrens- und Kunststofftechnik sowie aller Teilbereiche des Maschinenbaus. Ebenso wurde die Aufteilung nach Fern, Direktstudium und nach Aufbau- und Brückenkursen vernachlässigt. Das Angebot an letzteren war für einige Absolventinnen ein wichtiger Grund, sich nach 1991 in Wildau für ein Studium zu bewerben. Andere Hochschulen hatten diese Möglichkeiten nicht geboten.

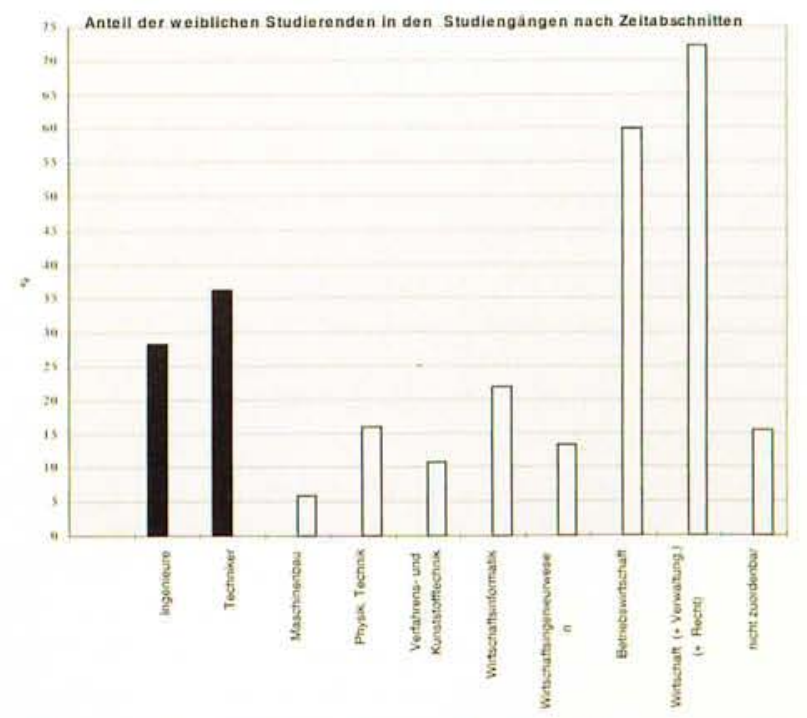

Graphik 1: Anteil der weiblichen Studierenden nach Fachrichtungen und Zeitabschnitten; Legende: blau 1984-1990, gelb 1991-1997; Quelle: Unterlagen der TFHW

\section{Ergebnisse in Kurzfassung}

\section{Population}

Grundlage der Befragung waren 286 Fragebögen, die an Betriebswirtinnen, Technikerinnen und Ingenieurinnen der Absolventenjahrgänge von 1987 bis 1997 versandt wurden. 50 Fragebögen waren unzustellbar (17,5 Prozent). Damit kann von einer erreichten Population von 236 Frauen ausgegangen werden, von denen 102 die Fragebogen ausgefüllt zurückgaben. Der Rücklauf von 43 Prozent ist als gut einzuschätzen. Die Ergebnisse sind statistisch nicht geprüft.

Entsprechend dem Rücklauf standen Angaben von Teilnehmerinnen zur Verfuigung, die zu 86 Prozent zwischen 22 und 33 Jahre jung sind, zu zwei Drittel in einer Partnerschaft leben, in knapper Mehrzahl keine Kinder haben, überwiegend in einer Festanstellung in Vollzeit arbeiten und ihr Studium mehrheitlich zwischen 1989/1990 bzw. 1995/1997 beendeten. Von den Probandinnen erwarben 25 einen akademischen Grad als Betriebswirtin, 23 als Technikerin, 20 in der Fachrichtung Maschinenbau, fünf als Konstrukteurin, eine als Physikerin. 11 Abschlüsse wurden auf anderen Gebieten erworben, meist außerhalb der Schule Wildau. 17 Angaben fehlen. 


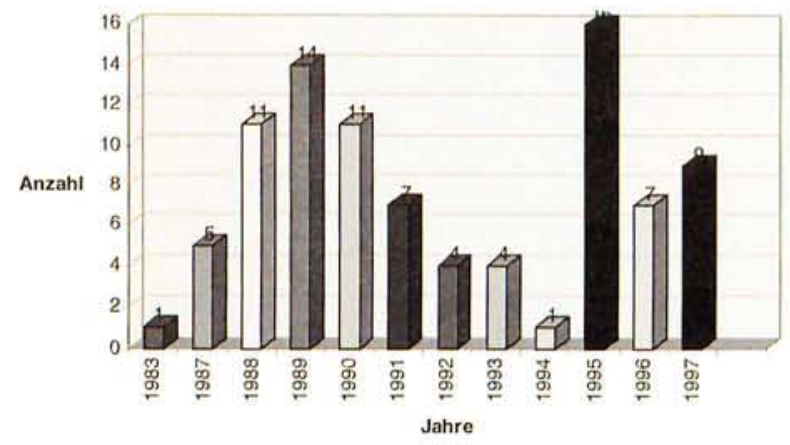

Graphik 2: Studienabschlïsse nach Abschlußjahren Quelle: Befragungsunterlagen

\section{Erwerbstätigkeit}

Erwerbstätig sind 87 Prozent der Probandinnen. 12 Prozent sind ohne Arbeit. Eine Absolventin ist selbständig tätig.

Von den 89 nichtselbständig Tätigen arbeiten 46 auf dem Gebiet ihres Studienfaches. Damit sind 51 Prozent der Absolventinnen gewissermaßen „Im Fach tätig“.

49 Prozent der Probandinnen arbeiten nicht auf dem Gebiet der studierten Fachrichtung. Als Ursachen (soweit genannt) können gelten: fünf Prozent persönliche /familiäre Gründe, 11 Prozent Interessenwandel nach dem Studium, 57 Prozent Mangel an Arbeitsplätzen, 11 Prozent Nichtanerkennung der Studienabschlüsse nach Einigungsvertrag. Zusammenfassend ergibt sich, daß die Gründe für eine Tätigkeit außerhalb der studierten Fachrichtung zu zwei Drittel den strukturellen und gesellschaftspolitischen Faktoren nach der Vereinigung der beiden Staaten zuzuordnen sind.

Von den 44 berufstätigen Frauen, die nicht im studierten Beruf arbeiten, würden 60 Prozent eine solche Chance gerne wahrnehmen. Daraus läßt sich eine hohe Identifikation mit der studierten Fachrichtung und wahrscheinlich auch ein vorangegangener ausgeprägter Studienwunsch ableiten.

\section{Beschäftigungsbranchen}

Die wichtigsten Beschäftigungsbereiche sind verarbeitendes Gewerbe (25,3 Prozent), Handel und Dienstleistungen (21,5 Prozent), Bau- und Baunebengewerbe (20,2 Prozent), Behörden und öffentliche Ämter $(15,1$ Prozent). Ca.17 Prozent arbeiten in Energie, Bergbau, Wasserwirtschaft, im Kredit- und Versicherungsgewerbe, in Kammern und Verbänden, im Verkehrswesen. Die Land-, Forst- und Fischwirtschaft sind keine Beschäftigungsbranchen fuir die Probandinnen. Insgesamt lagen 79 Angaben vor.

Innerhalb des verarbeitenden Gewerbes konzentrieren sich die Einsatzgebiete auf funf Bereiche. Hierbei sind der Maschinen- und Fahrzeugbau, die Herstellung von Büromaschinen und EDV-Anlagen mit 42 Prozent Anteil und der Bereich Elektrotechnik, Feinmechanik, Optik mit 23 Prozent Anteil der Arbeitsstellen die wichtigsten Branchen. Holz/Papier/Druck sowie Ernährung und Tabak sind mit je 9 Prozent vertreten

4. Unternehmensgröße und Beschäftigungsstruktur Kleine und mittlere Unternehmen mit zwei bis 199 Mitarbeitern stellen den Hauptanteil der Arbeitgeber für die
Probandinnen (55 Prozent). Innerhalb dieser Größengruppe ist eine Konzentration auf die kleinen Unternehmen bis 19 Beschäftigte (21 Prozent) und die größeren Betriebe von 50 bis 199 Beschäftigten (23 Prozent) festzustellen. Die mittlere Gruppe der Unternehmen mit 20 bis 49 Arbeitnehmern beschäftigt einen relativ kleinen Anteil der Absolventinnen (11 Prozent). 13 Prozent sind in Großbetrieben von tausend und mehr Mitarbeitern eingesetzt. Am geringsten ( 8 Prozent) ist der Anteil in der Unternehmensgröße zwischen 200 und 1000 Beschäftigte. 82 Angaben lagen vor.

Die Struktur der Beschäftigung zeigt Tabelle 2.

\section{TABELLE 2: Struktur der Erwerbstätigkeit}

\begin{tabular}{lc} 
Art der Erwerbstätigkeit & in Prozent \\
\hline Festanstellung Vollzeit & 75 \\
Festanstellung Teilzeit & 6 \\
keine Angaben & 15 \\
Sonstiges & 4 \\
\hline
\end{tabular}

Quelle: Eigene Befragungsunterlagen

Sonstige Arten von Erwerbstätigkeit erfassen Drittmittelstellen und Honorararbeiten u. ä.

\section{Beschäftigungsregionen}

Berlin und Brandenburg sind nicht nur bedeutende Einzugsgebiete der Bildungseinrichtung, sondern auch wichtige Beschäftigungsregionen für ihre Absolventinnen. 22 Prozent der Probandinnen arbeiten in Berlin und 17 Prozent in Brandenburg, 16 Prozent in Sachsen. Sieben Prozent sind in Thüringen beschäftigt. Die Länder Sachsen-Anhalt, Mecklenburg-Vorpommern sind mit zwei und vier Prozent vertreten,

Von den 102 Probandinnen arbeiten 14 (13,7 Prozent) in den alten Bundesländern, davon für Hessen, BadenWürttemberg, Niedersachsen je eine Angabe, für Hamburg zwei Angaben, für Nordrhein-Westfalen vier und für Bayern fünf Angaben. Acht der genannten Absolventinnen in den alten Bundesländern schlossen ihr Studium nach der Neugründung der Technischen Fachhochschule Wildau als Betriebswirtin (sieben) und als Maschinenbauerin (eine) ab.

\section{Berufsziele und Lebenserwartungen}

Hinsichtlich der Ziele des beruflichen Wirkens steht der Wunsch nach einer Arbeit, bei der Beruf und Familie miteinander verbunden werden können, bei einem Fünftel der Probandinnen an erster Stelle. 14 Prozent stellen sich das Ziel, beruflich aufzusteigen. 12 Prozent wünschen sich mehr Eigenverantwortung. Sieben Prozent sind mit ihrem beruflichen Leben zufrieden, haben keine Wünsche. 47 Prozent beantworteten die Frage nicht. Allerdings werden trotz des hohen Beschäftigungsgrades die künftigen beruflichen Chancen für Ingenieurinnen und Technikerinnen nicht positiv eingeschätzt. Hierbei geben die „Im Fach Tätigen“ Probandinnen (46 Personen), den beruflichen Aussichten von Ingenieurinnen und Technikerinnen etwas bessere Noten als die übrigen berufstätigen Probandinnen. Eine größere Gruppe 
von ihnen fühlt sich allerdings auch in der Einschätzung der Zukunftsaussichten deutlich unsicherer als die nicht im Studienfach tätigen Absolventinnen. Dieser scheinbare Widerspruch bedarf der näheren Untersuchung.

\section{Berufstätigkeit und Studium}

Als interessante Frage erschien die Bewertung des absolvierten Studiums, die nach verschiedenen Gesichtspunkten erfolgen sollte. Das Gesamtergebnis ist erwartungsgemäß nach der Gruppe der ,im Fach Tätigen“ und der „nicht im Fach Tätigen“ differenziert. Die Hälfte der Teilnehmerinnen äußerte sich nicht.

Bezogen auf die Gesamtheit der Probandinnen stellten nur 15 Prozent eine Übereinstimmung zwischen Ausbildungsinhalten und den Anforderungen der gegenwärtigen beruflichen Tätigkeit fest. 29 Prozent gaben keine eindeutige Stellungnahme ab, 5 Prozent verneinten eine solche Übereinstimmung.

In der Gruppe der im Studienfach tätigen Probandinnen stellten 34 Prozent eine Übereinstimmung zwischen Ausbildungsinhalten und den Anforderungen der gegenwärtigen beruflichen Tätigkeit fest. 60 Prozent gaben keine eindeutige Stellungnahme ab. 4 Prozent verneinten eine solche Übereinstimmung

Ein deutlicher Unterschied bei der Bewertung der Studieninhalte nach Altersgruppen ergab sich nicht.

\section{Bedürfnisse nach frauenspezifischen Lehrveranstaltungen}

Frauenspezifische Lehrveranstaltungen wurden von der Hälfte der Probandinnen abgelehnt. Ausbildung sollte nicht geschlechterspezifisch geprägt sein. Nur ein Füinftel bejahte sie eindeutig. Ein Drittel hatte sich nicht mit dem Problem beschäftigt bzw. gaben keine Antwort.

Zustim mung oder Ablehnung frauenspezifischer Lehrveranstaltungen sind

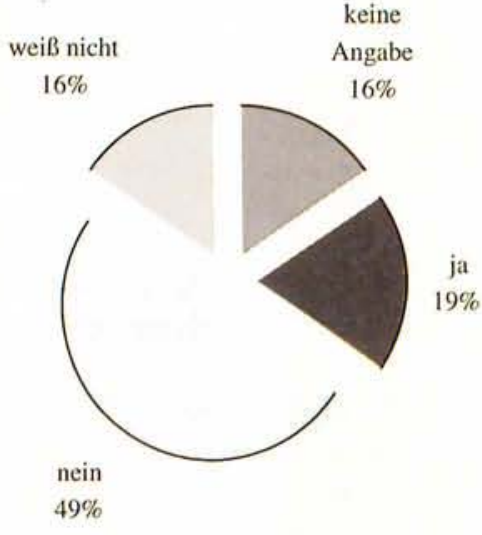

Graphik 3: Bedürfnis nach frauenspezifischen Lehrveranstaltungen. Quelle: Befragungsunterlagen in der Gesamtheit der Probandinnen durch die beiden jüngeren Altersgruppen 22 bis 27 bzw. 28 bis 33 Jahre dominiert. Beide Gruppen stellen den größten Anteil an den Befragungsteilnehmerinnen. Die Zustimmung wird zu 61 Prozent von der jüngsten Altersgruppe geprägt, die Ablehnung zu 58 Prozent von der Altersgruppe 28 bis 32 Jahre.

Innerhalb der einzelnen Altersgruppen verteilen sich Zustimmung und Ablehnung deutlich differenzierter. In der Altersgruppe 22-27 Jahre sprachen sich etwa ein Viertel der Absolventinnen für frauenspezifische Lehrveranstaltungen aus, in der (zahlenmäßig kleinen) Altersgruppe 33-39 Jahre sogar fast 34 Prozent. Mit ca. 10 Prozent Zustimmung war der Anteil in der Altersgruppe 2832 Jahre überraschend niedrig.

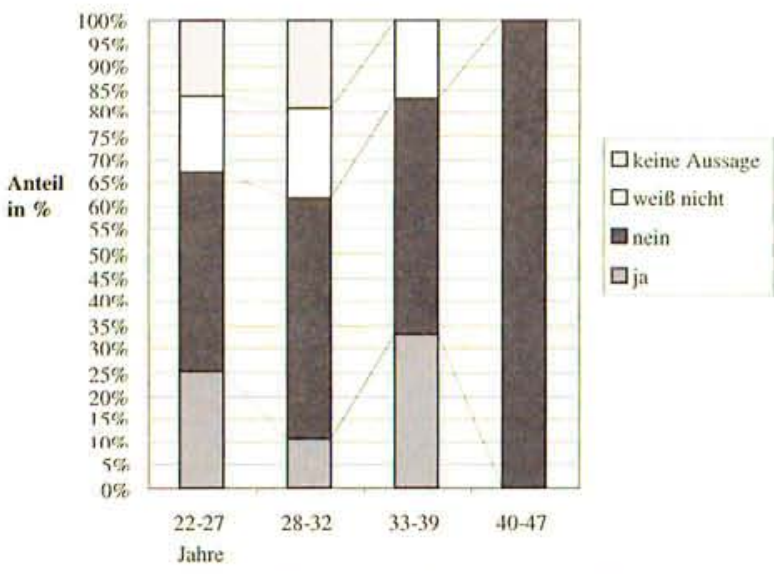

Graphik 4: Positionen zu frauenspezifischen Lehrveranstaltungen in den Altersgruppen (Prozent); Quelle: Befragungsunterlagen

\section{Fazit}

Die Befragung ergab interessante, zum Teil nicht erwartete Ergebnisse hinsichtlich der Einbindung von Absolventinnen der Ingenieurschule Wildau und der Technischen Fachhochschule Wildau auf dem Arbeitsmarkt. Schlußfolgerungen für die Ausbildung bedürfen der weiteren Diskussion. Tiefeninterviews mit ausgewählten Probandinnen, insbesondere der technischen Fachrichtungen könnten die Ergebnisse präzisieren.

\section{* Literaturhinweis}

Auf die Literatur zu diesen Fragen soll nicht eingegangen werden. Einige Hinweise auf Neueres:

- Tilo Görl, Beschäftigungssituation Brandenburger Frauen. Universität Potsdam, Wirtschafts- und Sozialwissenschaftliche Fakultät. MS September 1997.

- Hochschul-Informations-System GmbH. Berufliche Integration und Weiterbildung von Ingenieurinnen aus den neuen Ländern Reihe: Aktuell, Bildung, Wissenschaft, 3/93. BMBW, Bonn

- Wolfram Kempe, Hohe Erwerbsbereitschaft ostdeutscher Frauen unabhängig vom Einkommensniveau, in: Wirtschaft im Wandel, 1/1998

- Doris Jahnsen, Die Technische Universität der Frauen Europas. Eine konkrete Vision für die Jahrtausendwende., in: Frauen gestalten den Strukturwandel. Engagement von Frauen im technischen Bereich. Internationale Fachkonferenz unter Schirmherrschaft der Deutschen UNESCOKommission, Leipzig, 25. und 26. Januar 1996, Band 1, Leipzig 1996

\section{Verfasser}

Prof. Dr. sc. oec. Michael Laschke

Technische Fachhochschule Wildau

Fachbereich Betriebswirtschaft/Wirtschaftsinformatik Tel. (0 33 75) 508-910

Dr. phil. Brigitte Lindert

Berufsfortbildungswerk GmbH (bfw) Sachsen

Bildungsstätte, 04579 Espenhain

Tel. (03 41) 3011057 